\title{
Adaptação Brasileira do Manual Brief Intervention for Adolescent Alcohol and Drug Use ${ }^{1}$
}

\author{
Fernanda Cerutti, Bruna Fernandes da Rocha, Luísa Raquel Bridi Dacroce, Irani Iracema de Lima Argimon \\ Pontifícia Universidade Católica do Rio Grande do Sul, Porto Alegre-RS, Brasil
}

\section{RESUMO}

O objetivo deste estudo é apresentar o processo de adaptação para a realidade brasileira do Manual Brief Intervention for Adolescent Alcohol and Drug Use. Na versão original, desenvolvida nos Estados Unidos, o formato das sessões é individual, sendo assim, também se objetivou adaptar o manual para o formato grupal. O protocolo prevê a realização de quatro sessões, sendo que duas delas contam com a participação dos pais/responsáveis. O processo foi realizado em três etapas: 1. tradução e retrotradução do manual e a adaptação das intervenções e recursos; 2. revisão do material por especialistas; 3. adequação das intervenções por meio de dois estudos-piloto, o primeiro em formato individual e o segundo em formato grupal. Os resultados evidenciaram a adequação da versão do manual para a realidade brasileira e para ser utilizado em grupos.

Palavras-chave: adolescência, intervenção breve, uso de drogas, treinamento de pais.

\section{ABSTRACT - Brazilian Adaptation of the Brief Intervention for Adolescent Alcohol and Drug Use Manual}

The purpose of this study is to present the adaptation process of the Brazilian version of the manual Brief Intervention for Adolescent Alcohol and Drug Use. In the original version, developed in the United States, the session format is individual, and so, another objective was to adapt the manual for the group format. The protocol provides for four sessions, two of which involve participation of parents/guardians. The process was carried out in three stages: 1. translation and back-translation of the manual and adaptation of interventions and resources; 2. expert review of the material; 3. verification of intervention adequacy through two pilot studies, the first in an individual format and the second in a group format. The results evidenced the adequacy of the manual revision for the Brazilian context and for use in groups.

Keywords: Adolescence; Brief Intervention; Use of drugs; Parent Training.

\section{RESUMEN - Adaptación Brasileña del Manual Brief Intervention for Adolescent Alcohol and Drug Use}

El objetivo de este estudio es presentar el proceso de adaptación en el contexto brasileño del Manual Brief Intervention for Adolescent Alcohol and Drug Use. En la versión original desarrollada en Estados Unidos, el formato de las sesiones es individual, siendo así, el objetivo del trabajo también fue adaptar el manual para el formato grupal. El protocolo incluye cuatro sesiones, dos de ellas con la participación de los padres/tutores. El procedimiento se llevó a cabo en tres etapas: 1. traducción y retro-traducción del manual y adaptación de intervenciones y recursos; 2. revisión del material por especialistas; 3. adecuación de las intervenciones por medio de dos estudios piloto, el primero en formato individual y el segundo en formato grupal. Los resultados señalaron adecuación de la versión del manual para la realidad brasileña y para ser utilizado en grupos.

Palabras clave: Adolescencia; Intervención Breve; Uso de drogas; Entrenamiento de padres.

\section{Introdução}

Atualmente, há tratamentos e intervenções baseadas em evidências que se mostraram efetivos para adolescentes com uso problemático de drogas (National Institute for Abuse [NIA], 2014). Dentre esses tratamentos, estão as intervenções breves, cujo foco é a motivação do adolescente para a mudança no seu hábito de uso de drogas. Sendo essa uma alternativa adequada para intervir precocemente e preventivamente com esse público (Winters, Leitten, Wagner, \& Tevyaw, 2007). Elas são convenientes para os profissionais da saúde pela rápida aplicação, baixo custo, efetividade e por serem clinicamente aplicáveis a quase todos os usuários de substâncias de grau leve a moderado (Winters, Fahnhorst, Botzet, Lee, \& Lalone, 2012; Winters, Tanner-Smith, Bresani, \& Meyers, 2014).

No Brasil, essa temática vem sendo destacada com maior ênfase pela equipe de pesquisadores da Faculdade de Medicina de Botucatu/Unesp (Faria, 2016). A equipe está desenvolvendo estudos utilizando o Brief Alcohol Screening and Intervention for College Students - BASICS (Dimeff, Baer, Kivlahan, \& Marlatt, 2002), que consiste

Agradecimentos: Ao apoio financeiro das agências de fomento CNPq, CAPES e FAPERGS. Às psicólogas Ms. Fernanda de Almeida Ribeiro e Dr. Martha Wallig Brusius Ludwig pela participação como juízas do manual e aos auxiliares de pesquisa do Grupo Avaliação e Intervenção no Ciclo Vital (PUCRS) que realizaram a tradução do material. Assim como, um agradecimento especial aos adolescentes e seus responsáveis que colaboraram com os estudos-piloto. E aos locais que permitiram a realização da intervenção e indicaram os participantes.

Endereço para correspondência: E-mails: fernanda.cerutti@imed.edu.br; fernanda.cerutti@acad.pucrs.br 
em uma intervenção breve para estudantes, focalizado no uso problemático de álcool, e que acontece em dois encontros com intervalo de 15 dias, com duração de 50 minutos (Silva \& Tucci, 2015).

Com o avanço de estudos de intervenção, é necessário que alguns pontos sejam cuidadosamente levados em consideração, destaca-se a atenção a intervenções direcionadas ao grau de gravidade do uso da substância, com foco no prolongamento dos resultados alcançados, nos fatores mediadores e moderadores de comprometimento no processo de mudança comportamental, bem como no papel dos pais na aderência ao tratamento e no apoio na recuperação (Winters et al., 2012; Winters et al., 2014).

Sobre a participação dos pais no tratamento do filho usuário de substâncias psicoativas, a literatura apresenta uma influência direta destes no uso problemático do adolescente. Algumas atitudes são frequentemente discutidas, como a permissão, oferecimento e supervisão dos pais no uso de álcool do filho em casa, que facilitam a aderência dos adolescentes às bebidas antes da maioridade (Kaynak, Winters, Cacchiola, Kirby, \& Arria, 2014). Mesmo essas atitudes se tornando um fator de risco para o uso, a literatura mostra que a motivação parental para estimular o uso de álcool do adolescente em casa se dá principalmente para evitar um papel desagradável ao filho que está sendo pressionado pelos pares (Wadolowski et al., 2016). Esse comportamento indica a necessidade dos pais de receberem orientações profissionais.

Entretanto, apesar de se reconhecer a importância dos pais na aderência de seus filhos ao uso de substâncias, sabe-se que esse assunto ainda é pouco contemplado no tratamento usual do adolescente usuário de drogas. Em um estudo que buscou analisar serviços de tratamento, focando na existência do trabalho de habilidades parentais, identificou-se que somente $6 \%$ dos programas oferecidos trabalhavam habilidades parentais específicas em sessões individuais, sendo que $3 \%$ abordavam o tema dentro de um cronograma exclusivo (Arria et al., 2013).

Sabendo da necessidade de incluir os pais/responsáveis de forma mais efetiva no tratamento do adolescente envolvido com álcool e ou outras drogas, Winters et al. (2006) desenvolveram um manual de tratamento de intervenção breve que contempla sessões de psicoeducação e treinamento para pais/responsáveis, e o nomearam de Brief Intervention for Adolescent Alcohol and Drug Use. Os autores testaram empiricamente esse protocolo de intervenção.

A primeira versão do manual foi desenvolvida por Winters e Leitten (2001). Alguns ajustes no manual foram efetivados em um primeiro ensaio clínico randomizado realizado com 79 adolescentes, identificados no contexto escolar pelo consumo de drogas. Os participantes foram divididos em três grupos: 1 . somente com o adolescente; 2. com adolescente e pais; e 3. grupo controle. Nessa primeira aplicação para testar a efetividade, o grupo com adolescentes e pais foi o que demonstrou melhores resultados (Winters \& Leitten, 2007).

A Brief Intervention for Adolescent Alcohol and Drug Use então foi novamente testada em um ensaio clínico randomizado mais expandido, sendo aplicada em 315 adolescentes de 12 a 18 anos, os quais foram identificados no ambiente escolar pelo uso de substâncias psicoativas. Seguindo os mesmos três grupos do estudo de Winters e Leitten (2007), os resultados mostraram que o grupo em que somente os adolescentes recebiam intervenção e aquele em que os adolescentes e seus pais recebiam apresentaram resultados significativos de redução do comportamento do uso de substâncias em comparação ao grupo controle. O grupo com adolescentes e pais apresentou melhores resultados em diversas variáveis (resolução de problemas, estratégias de enfrentamentos e utilização dos serviços de aconselhamentos) quando comparado ao grupo com somente os adolescentes (Winters et al., 2012).

No estudo de Winters et al. (2014), foi realizado o follow-up 12 meses após a intervenção dessa mesma amostra, participando 284 adolescentes (Winters et al., 2012). Os resultados apontaram que os ganhos positivos da intervenção permaneceram ao longo do tempo. Outro resultado encontrado foi a diminuição significativa do uso de cannabis nos grupos de intervenção comparado ao grupo-controle.

A intervenção breve com sessões com pais também foi aplicada no estudo de Dembo et al. (2014) com 166 adolescentes de 11 a 15 anos usuários de maconha e que apresentavam comportamentos sexuais de risco. Os resultados demonstram que a intervenção não gerou efeitos significativos sobre ambos os comportamentos de risco ao longo do tempo, em medidas follow-up que aconteceram três, seis e 12 meses após a intervenção. Os autores discutiram o número reduzido de participantes para a realização das análises e a influência de outras variáveis no resultado. Contudo, a aplicação da intervenção breve com pais/responsáveis com foco em outros fatores de risco associados ao uso de drogas, destaca a importância desse protocolo.

Com isso, este estudo objetivou descrever o processo de adaptação para a realidade brasileira do manual Brief Intervention for Adolescent Alcohol and Drug Use. Especificamente, apresentar as fases de 1. tradução, retrotradução e adaptação, 2. revisão de especialistas e 3. teste da adequação da aplicabilidade clínica (estudo piloto 1), bem como da aplicação do manual na versão grupal (estudo piloto 2). Para esse último objetivo, foi utilizada como medida o grau de motivação para o tratamento e prontidão para a mudança de comportamento. Entende-se que o construto da motivação é um fator muito importante para o tratamento de adolescentes (Maisto, Chung, Cornelius, \& Martin, 2003) e que sofre muita influência com a participação dos pais no tratamento (Vanassche et al. 2014). 


\section{Método}

O processo de realização de um estudo de adaptação transcultural de instrumentos clínicos, de acordo com a literatura (Hungerbühler \& Wang, 2016), requer o cumprimento de algumas fases: (1) definições conceituais; (2) tradução; (3) retrotradução; (4) revisão por especialistas; (5) estudo piloto; e (6) testes psicométricos para validação. Assim, o processo de tradução e adaptação do manual Brief Intervention for Adolescent Alcohol and Drug Use (Winters et al., 2006) foi dividido em três etapas: 1. tradução do manual para o português brasileiro, retrotradução para o inglês norte-americano e adaptação para realidade brasileira, 2. análise de juízes da adequação da tradução; e 3. estudo piloto 1 e piloto 2 com amostra clínica para teste de aplicabilidade, tanto da adaptação do manual, quanto do formato grupal. A seguir são descritos os procedimentos realizados em cada uma dessas etapas.

\section{Apresentação da Brief Intervention for Adolescent Alcohol and Drug Use}

A Intervenção Breve proposta no manual consiste em quatro sessões estruturadas, com duração prevista de $1 \mathrm{~h}$ a $1 \mathrm{~h}$ e meia, em formato individual - acompanha somente um adolescente e seus pais/responsáveis. Os intervalos entre as sessões são de sete dias entre as três primeiras sessões, e a quarta e última é após um mês. Os objetivos das sessões são:

$1^{a}$ Sessão: realizada somente com o adolescente, visa identificar os eventos que causam o uso, examinar crenças ligadas a esses eventos de ativação e desenvolver crenças funcionais ligadas à abstinência.

Nessa sessão, está prevista a utilização das seguintes técnicas:

- Questionário para o Adolescente: investiga sobre o uso de drogas e sobre o reconhecimento do problema relacionado a esse uso;

- Planilha de Vantagens e Desvantagens: trata-se de uma exploração e discussão sobre as consequências positivas e negativas do uso de drogas;

- Planilha de Gatilhos e Fissura: apresenta uma lista de razões comuns que podem levar o adolescente ao uso ou a lapsos;

- Coisas que fazem você feliz: Uma lista com várias atividades que não envolvem o uso de drogas, mas que podem ser opções que trazem igualmente prazer e que podem ser protetivas;

- Planilha: O que desencadeia o seu uso de álcool e/ou outras drogas: em uma lista, deve-se colocar as situações-gatilho anteriormente analisadas e as alternativas para não usar, também já trabalhadas;

- Situações de gatilhos e fissura: algumas situações comuns que causam emoções desagradáveis são expostas e é solicitado que o adolescente pense em como reagiria no passado e o que poderia fazer no momento presente, após o que foi discutido;

- Planilha Prontidão para a mudança (Parte I): avaliar, por meio de uma régua de prontidão para mudança, como está se sentindo naquele momento.

$2^{a}$ Sessão: com pais/responsáveis, busca discutir a importância da intervenção, desenvolver atitudes parentais eficazes, examinar as atitudes pessoais sobre o uso de drogas e definir e incentivar o monitoramento. Na sessão 2, está previsto o uso das seguintes técnicas:

- Regras da família sobre o uso de drogas: discutir sobre as regras e atitudes familiares sobre o uso de drogas.

- Planilha Pais/Responsáveis: para analisar sobre o monitoramento e a supervisão parental.

- Fatores de risco e de proteção: recurso para psicoeducar os cuidadores sobre fatores que aumentam o risco para uso de drogas.

$3^{a}$ Sessão: com adolescente, objetiva focar na resolução de problemas, no desenvolvimento de métodos, componentes e habilidades para lidar com o uso de drogas.

- Técnicas de Recusa: diferentes tipos de respostas para dizer "não" e recusar as ofertas de drogas.

- Planilha de apoio social: recurso para discutir com o adolescente sobre quem pode ser um apoio para um estilo de vida sem o uso de drogas.

- Planilha de Prontidão para a Mudança (Parte II): investiga os objetivos do adolescente em mudar seu comportamento de uso de drogas em seis meses e no próximo mês e retoma a mesma régua de prontidão para mudança da sessão 1.

$4^{a}$ Sessão: com pais/responsáveis e adolescentes, foca na análise da interação entre pais/responsáveis e adolescentes, incentivando a comunicação eficaz e um bom relacionamento familiar. Realiza-se a "prestação de contas" dos objetivos da intervenção.

- Questionário: Concordância sobre as atitudes a favor das mudanças: é solicitado que adolescentes e responsáveis analisem o esforço para mudança em si e no outro.

- Seis etapas para comunicação eficaz: trabalha pontos importantes para desenvolver uma comunicação eficaz em situações estressantes.

- Como melhorar sua argumentação: trabalha atitudes para argumentar seu ponto de vista de uma forma mais hábil.

\section{Etapa 1: Tradução, Retrotradução e Adaptação do Manual}

O manual foi traduzido para a língua portuguesa por seis graduandos de psicologia proficientes em inglês. A retrotradução consistiu na realização da tradução novamente para o inglês norte-americano dos materiais e recursos das sessões que foram traduzidos para o português brasileiro. Essa etapa de retrotradução realizada por uma psicóloga com conhecimento das terminologias e conceitos 
apresentados no manual e com imersão na língua inglesa e uma graduanda de psicologia de uma universidade norte-americana. Posteriormente, foi realizada a adaptação das expressões e intervenções do manual para a realidade brasileira por uma doutoranda proficiente em inglês e com conhecimento das teorias e técnicas descritas no manual.

\section{Etapa 2: Revisão por Especialistas da Equivalência Semântico-Idiomática e Cultural}

Nessa etapa, de avaliação da tradução e adaptação do manual, participaram dois juízes, ambos psicólogos, que possuem conhecimento na língua inglesa e nas teorias que embasam a intervenção e com experiência no trabalho com adolescentes. A tarefa deles foi realizar a análise do documento traduzido, focando na terminologia e conceitos da fundamentação teórica e nas expressões e instruções de cada sessão. Foi enviado aos juízes um documento solicitando que preenchessem sobre a adequabilidade dos principais itens do manual, como pode ser observado na Figura 1.

\begin{tabular}{lll}
\hline \multicolumn{1}{c}{ Itens analisados } & \multicolumn{1}{c}{ Adequabilidade } \\
\hline Fundamentação Teórica (Terminologia e conceitos) & $\begin{array}{l}\text { ( ) Adequado } \\
\text { Sugestões: }\end{array}$ \\
Sessão 1 (Expressões e Instruções) & $\begin{array}{l}\text { ( ) Adequado } \\
\text { Sugestões: }\end{array}$ & ( ) Inadequado \\
Sessão 2 (Expressões e Instruções) & $\begin{array}{l}\text { ( ) Adequado } \\
\text { Sugestões: }\end{array}$ \\
Sessão 3 (Expressões e Instruções) & $\begin{array}{l}\text { ( ) Adequadequado } \\
\text { Sugestões: }\end{array}$ \\
Sessão 4 (Expressões e Instruções) & $\begin{array}{l}\text { ( ) Adequadequado } \\
\text { Sugestões: }\end{array}$ \\
\hline
\end{tabular}

Figura 1. Instrumento para avaliar a adequabilidade da tradução e adaptação do manual

\section{Etapa 3: Teste de Aplicabilidade}

No estudo piloto 1 , as sessões e intervenções foram testadas a partir da aplicação em um adolescente e seus pais. Nessa etapa, verificou-se a adequação das intervenções para a realidade brasileira, além do tempo de duração das sessões. Manteve-se o formato individual, da mesma forma que o manual original.

No estudo piloto 2, objetivou-se testar as intervenções e as sessões no formato grupal; participaram dele 10 adolescentes e seus responsáveis. As sessões foram conduzidas em grupo, tanto com os adolescentes, quanto com os pais. O objetivo do estudo piloto 2 foi verificar se a realização das sessões previstas no manual necessitava de ajustes ao serem aplicadas em grupo.

\section{Seleção dos Participantes}

No estudo piloto 1 , o adolescente, indicado por um ambulatório especializado em tratamento da dependência química, foi escolhido segundo critérios estabelecidos para a seleção dos participantes do estudo: a) faixa etária dos 13 aos 17 anos; b) uso de álcool e/ou outra droga; c) ausência de sintomas psicóticos graves ou outro transtorno clínico que impedisse sua participação e compreensão das intervenções; e d) consentimento dos pais para a participação do adolescente no estudo e sua concordância em participar.
No estudo piloto 2, participaram 10 adolescentes e seus responsáveis. Eles foram escolhidos por conveniência e também fecharam os critérios estabelecidos para seleção dos participantes, que foram citados anteriormente. Esses participantes estavam internados em um centro de tratamento de adolescentes com problemas com o uso de drogas.

\section{Medida de Pré-Teste e Pós-Teste}

O teste da compreensão das intervenções traduzidas (estudo piloto 1) e da viabilidade da aplicação grupal (estudo piloto 2) foi realizado por meio de uma medida de grau de motivação e de prontidão para a mudança do comportamento de uso de drogas. Para tanto, foi utilizada a Escala de Contemplação Ladder. Esse instrumento é uma medida breve de motivação e de prontidão para a mudança. Foi desenvolvido por Biener e Abrams em 1991, e, no Brasil, Terra et. al (2009) realizaram uma validação convergente para a população de tabagistas.

Neste estudo, foi utilizada uma versão com cinco afirmativas que avaliam, não só o uso de tabaco, mas das demais classes de drogas. Os adolescentes devem escolher entre as afirmativas: 1 - "Não penso em parar de usar droga/álcool/tabaco"; 2 - "Penso que será necessário parar algum dia"; 3 - "Penso em parar, mas ainda não estou pronto"; 4 - "Estou começando 
a pensar sobre como mudar meu hábito de usar droga/ álcool/tabaco"; e 5 - "Estou fazendo alguma coisa para parar de usar droga/álcool/tabaco". Aqueles que se avaliam próximos ao número 1 , ainda se encontram pré-contemplativos. Já avaliações próximas ao cinco remetem ao estágio da ação (Amodei \& Lamb, 2004; Terra et. al, 2009).

\section{Considerações Éticas}

A tradução e a adaptação do manual foram autorizadas por e-mail pelo autor responsável, Professor Dr. Ken C. Winters. O estudo foi aprovado pelo Comitê de Ética de Pesquisa local (CAAE: 48681015.6.0000.5336). Após a indicação e aceite dos participantes, os pais assinaram um Termo de Consentimento Livre e Esclarecido concordando em participar do estudo, e outro permitindo a participação do adolescente. Os adolescentes, por sua vez, assinaram um Termo de Assentimento, explicitando sua disposição em participar do estudo. Todos os adolescentes que participaram do estudo seguiram em tratamento após a participação na pesquisa.

\section{Resultados}

Os resultados das etapas 1 e 2 serão apresentados de forma qualitativa e foram fundamentados nas anotações em documentos padronizados. Os dados da terceira etapa serão apresentados de forma descritiva (frequência, médias e desvio padrão) e a partir de pontuações diretas. Assim como, de forma qualitativa, de acordo com informações baseadas em anotações no diário de campo.

\section{Etapa 1: Tradução, Retrotradução e Adaptação do Manual}

A partir da Etapa de Tradução do Manual, optou-se por traduzir também o nome do protocolo de intervenção, no original Brief Intervention for Adolescent Alcohol and Drug Use: Manual. O nome na versão brasileira ficou
"Intervenção Breve para Adolescentes e Pais - (IBAP): Manual para tratar o uso de álcool e outras drogas na adolescência". Na tradução do nome, optou-se por fazer menção a participação dos pais e por incluir-se uma abreviatura, para facilitar a identificação dessa intervenção na versão brasileira.

No processo de retrotradução, observou-se que alguns termos da versão original em inglês tinham sido traduzidos por termos mais usuais no português brasileiro. Na retrotradução, houve divergências quanto a tradução dos termos "pros and cons", como visto na versão original do manual. Na tradução ficou "vantagens e desvantagens" e na retrotradução advantages and disadvantages. Com essa discrepância, decidiu-se por deixar a tradução para "vantagens e desvantagens" em todo o material, entendendo-se que seria melhor compreendida no português e acessível ao público que se quer atingir.

$\mathrm{Na}$ adaptação dos termos e recursos, destaca-se as alterações realizadas no material "Coisas que me fazem feliz”, que lista possíveis atividades prazerosas que não incluem o uso de drogas. Observou-se que, como a lista original continha atividades que eram populares entre adolescentes de uma década atrás (ex.: buying $C D$; writing in a diary or writing letters), optou-se por se incrementar a lista com atividades atuais (ex.: baixar músicas; usar redes sociais).

\section{Etapa 2: Revisão por Especialistas da Equivalência Semântico-Idiomática e Cultural}

A análise de adequação da tradução e equivalência semântico-idiomática e cultural realizada por dois juízes demonstrou necessidade de poucas alterações quanto ao conteúdo em sua totalidade. Houve sugestões em cada um dos itens avaliados, com ênfase nos que apresentaram dificuldades na compreensão da tradução e equívocos na tradução de termos. A Tabela 1 apresenta algumas sugestões apontadas pelos juízes.

Tabela 1

Apontamentos dos Juízes sobre Equivalência Semântico-Idiomática de Alguns Itens do Manual

\begin{tabular}{|c|c|c|c|}
\hline Item avaliado & $\begin{array}{l}\text { Versão Original } \\
\text { (Inglês) }\end{array}$ & $\begin{array}{l}\text { Tradução } \\
\text { (Português) }\end{array}$ & $\begin{array}{l}\text { Sugestões } \\
\text { (Juízas) }\end{array}$ \\
\hline \multirow[t]{4}{*}{$\begin{array}{l}\text { Fundamentação } \\
\text { teórica }\end{array}$} & Users of Brief Intervention & $\begin{array}{l}\text { Aplicadores da Intervenção } \\
\text { Breve }\end{array}$ & $\begin{array}{l}\text { Quem pode aplicar a } \\
\text { Intervenção Breve? }\end{array}$ \\
\hline & $\begin{array}{l}\text { Clients for whom Brief } \\
\text { Intervention is intended }\end{array}$ & $\begin{array}{l}\text { Clientes para os quais se } \\
\text { destina a Intervenção Breve }\end{array}$ & $\begin{array}{l}\text { A quem se destina esta } \\
\text { Intervenção Breve? }\end{array}$ \\
\hline & Potential Setting & $\begin{array}{l}\text { Setting de aplicação } \\
\text { da intervenção }\end{array}$ & $\begin{array}{l}\text { Possíveis locais de } \\
\text { aplicação da intervenção }\end{array}$ \\
\hline & ...encouraging self-efficacy & ...encorajar a autoeficácia & ...promover a autoeficácia \\
\hline \multirow[t]{2}{*}{ Sessão 1} & $\begin{array}{l}\text {...the beginning of } \\
\text { the stages of change }\end{array}$ & $\begin{array}{l}\text {...no início dos } \\
\text { estágios de mudança }\end{array}$ & $\begin{array}{l}\text {...nos estágios } \\
\text { iniciais de mudança }\end{array}$ \\
\hline & $\begin{array}{l}\text {...is the key to a } \\
\text { healthy lifestyle }\end{array}$ & $\begin{array}{l}\text {...é a chave para } \\
\text { uma vida saudável }\end{array}$ & $\begin{array}{l}\text {...é o caminho para } \\
\text { uma vida saudável }\end{array}$ \\
\hline
\end{tabular}


Tabela 1 (continuação)

Apontamentos dos Juízes sobre Equivalência Semântico-Idiomática de Alguns Itens do Manual

\begin{tabular}{|c|c|c|c|}
\hline Item avaliado & $\begin{array}{l}\text { Versão Original } \\
\text { (Inglês) }\end{array}$ & $\begin{array}{l}\text { Tradução } \\
\text { (Português) }\end{array}$ & $\begin{array}{c}\text { Sugestões } \\
\text { (Juízas) }\end{array}$ \\
\hline Sessão 2 & $\begin{array}{l}\text {... let's spend some } \\
\text { time talking about }\end{array}$ & $\begin{array}{l}\text {..vamos passar algum tempo } \\
\text { falando sobre }\end{array}$ & ...vamos falar um pouco sobre \\
\hline Sessão 3 & ...sobadly & ...lamento por você & ...que pena \\
\hline Sessão 4 & $\begin{array}{l}\text { Thanks for meeting with me } \\
\text { again today; I look forward } \\
\text { to hearing about how your } \\
\text { situation has changed over } \\
\text { the past month or so, and to } \\
\text { start discussing what types of } \\
\text { actions to take at this point. }\end{array}$ & $\begin{array}{l}\text { Obrigado por se encontrarem } \\
\text { comigo hoje de novo, estou } \\
\text { ansioso para ouvir sobre como } \\
\text { a situação de vocês evoluiu ao } \\
\text { longo deste mês que passou e } \\
\text { começar a discutir que tipos } \\
\text { de ações serão tomadas neste } \\
\text { momento. }\end{array}$ & $\begin{array}{l}\text { Obrigado por se encontrarem } \\
\text { comigo hoje de novo; eu gostaria } \\
\text { de ouvir como a situação de } \\
\text { vocês evoluiu ao longo deste } \\
\text { mês que passou, se evoluiu, } \\
\text { e começar a discutir que tipo } \\
\text { de ações serão tomadas neste } \\
\text { momento. }\end{array}$ \\
\hline
\end{tabular}

\section{Etapa 3: Teste de Aplicabilidade e da Versão Grupal: Estudos-Piloto}

\section{Caracterização dos Participantes}

O participante do estudo piloto 1 tinha 16 anos, e a média de idade dos participantes do estudo piloto 2 foi 16 anos $(\mathrm{DP}=0,82)$ - todos do sexo masculino. Especificamente, o adolescente do estudo piloto 1 apresentava uso problemático de álcool e os adolescentes do estudo piloto 2 eram poliusuários. $\mathrm{O}$ adolescente do estudo piloto 1 frequentava o ensino médio e a maioria dos adolescentes do estudo piloto 2 estava no ensino fundamental $(90 \%)$, sendo que um deles estava sendo alfabetizado.

Em relação à caracterização dos pais/responsáveis que participaram das sessões: no estudo piloto 1 , participaram o pai e a mãe e, no segundo piloto, a maioria foram mães $(n=7)$, e os outros três eram: pai, irmã e avó. Quanto ao uso de drogas, no estudo piloto 1, ambos os pais haviam feito uso de drogas ilícitas durante a vida. Já, no estudo piloto $2,40 \%$ dos responsáveis apresentaram uso problemático de tabaco e nenhum havia experimentado drogas ilícitas.

\section{Alterações Realizadas a partir do Estudo Piloto 1}

O estudo piloto 1 caracterizou-se pela realização do protocolo de intervenção com um adolescente e seus pais. Esse primeiro uso do manual adaptado para a realidade brasileira proporcionou que algumas questões, como a aplicabilidade das intervenções e o tempo de realização de todos os itens previstos no protocolo fossem testados e, sendo necessário, revistos.

$\mathrm{Na}$ sessão 1, não foi possível cumprir com todas as atividades previstas no manual em apenas um encontro com duração de uma hora - foi necessário agendar um encontro adicional. Assim, observa-se que essa primeira sessão com o adolescente deve ser realizada com a duração de 1 hora e 30 minutos. Não sendo possível disponibilizar esse tempo, sugere-se que o instrumento Questionário para o Adolescente possa ser aplicado em um encontro anterior destinado para uma entrevista, pois objetiva colher informações sobre a história do adolescente e sobre seu uso de drogas e, originalmente, já estaria prevista sua retomada durante a primeira sessão do protocolo.

No questionário Situações de Gatilhos ou Fissura, que é aplicado na Sessão 1, era solicitado que o adolescente respondesse às perguntas: "O que você faria? Existiria outra maneira de você lidar com esta situação?" e "Como você teria agido nesta situação no passado? O que você pode fazer agora, em vez de usar drogas e/ou álcool?”, pensando em cada uma das seguintes situações:

1. Você está em uma festa com seus amigos e alguém lhe passa um baseado. Você não sente vontade de fumar neste momento.

2. Você teve um dia muito difícil. Você tirou uma nota baixa na prova, seu melhor amigo(a) está afastado de você e você está se sentindo péssimo.

3. Você tem uma grande apresentação amanhã para um grande número de pessoas conhecidas. Você está muito nervoso e está tendo dificuldade para pegar no sono.

Após o piloto, houve a necessidade de reduzir o tempo de aplicação desse recurso, passando-se, então, a solicitar que o adolescente escolhesse uma dessas situações descritas, pensando naquela que mais está de acordo com a sua realidade e, em seguida, responder às perguntas (apresentadas anteriormente).

Na sessão 2, com os pais, como forma de também otimizar a utilização do tempo da sessão, observou-se que o instrumento Regras da Família sobre o Uso de Drogas, que trata sobre as atitudes ou regras familiares em relação ao uso de álcool e/ou drogas, continha perguntas muito extensas e que acabavam ficando repetitivas conforme iam sendo respondidas. Assim, optou-se por deixar as questões mais objetivas sem que o sentido fosse alterado, como, por exemplo, na versão pré-piloto, a questão 1 estava assim:

"Estudos têm mostrado a importância da criação de regras familiares ou de expectativas familiares sobre o uso de álcool e outras drogas para o filho(a) adolescente. Você 
tem regras ou expectativas sobre isso em sua casa? Quais os fatores que influenciaram a criação de regras/expectativas sobre o uso de drogas e álcool?"

Após a realização do piloto, a questão 1 ficou desta forma:

"Você tem regras sobre o uso de álcool e outras drogas em sua casa? Se sim, o que fez com que essas regras fossem estabelecidas?"

Ainda na sessão 2, percebeu-se que no instrumento Planilha Pais/Responsáveis, da mesma forma continha perguntas muito longas, fazendo com que os pais tivessem que reler muitas vezes cada uma. Com isso, verificou-se a necessidade de transformar as questões em um formato mais objetivo, para um melhor uso do tempo para discussão e algumas perguntas foram divididas em mais de uma questão para facilitar a compreensão do respondente.

Para ilustrar será apresentada a modificação em uma das questões, antes assim:

"Você pode me falar um pouco sobre os interesses/hobbies de seu filho(a)? Quais são os pontos fortes dele/dela? Quais são as coisas que são difíceis para ela/ele? (Tente descobrir qual tipo de atividade, em grupos, clubes, etc.)."

Depois, foi transformada em duas questões mais objetivas:

1. "Você tem conhecimento sobre as coisas que o(a) adolescente mais gosta ou se interessa? Se sim, conseguiria descrever algumas?

2. Você conseguiria descrever quais são os pontos fortes dele(a)? E quais são as coisas que são difíceis para ele(a)? Se sim, pode escrever aqui?

$\mathrm{Na}$ quarta e última sessão, em que estão presentes pais/responsáveis e adolescente, há um instrumento a ser preenchido por ambos para discutir mudanças de comportamento, tanto dos pais/responsáveis, quanto do adolescente. Ocorre que, na versão original, o instrumento: Questionário Concordância sobre as Atitudes a favor das Mudanças não distinguia quem estava respondendo, parecendo ser direcionado aos pais/responsáveis somente, enquanto o manual instrui que ambos - pais/responsáveis e adolescentes - expressem suas opiniões. $\mathrm{O}$ rapport do instrumento causou dúvidas aos respondentes na aplicação do piloto, levando à designação de uma versão para o adolescente e outra para os pais/responsáveis:

- Rapport do questionário (versão pais/responsáveis): "Vamos rever a forma como as coisas têm acontecido desde nosso último encontro. Estou interessado em saber do esforço do adolescente em melhorar aspectos específicos dos comportamentos saudáveis. Eu gostaria de pedir que cada um avalie a quantidade de esforço que o adolescente tem feito desde o nosso último encontro. Por favor, indique um número de 1 a 5 [...] Agora, é o momento de avaliar o esforço de vocês, pais ou responsáveis, para melhorar o... [Seguem as alternativas de respostas]".
- Rapport do questionário (versão adolescente): "Vamos rever a forma como as coisas têm acontecido desde nosso último encontro. Estou interessado em saber do esforço que você tem feito em melhorar aspectos específicos dos comportamentos saudáveis. Gostaria de pedir que você avalie a quantidade de esforço que tem feito desde o nosso último encontro. Por favor, indique um número de 1 a 5 [...]. Agora é o momento de avaliar o esforço dos pais ou responsáveis para melhorar o...[Seguem as alternativas de respostas]".

Na sessão 3, não houve modificações nos instrumentos e nas intervenções propostas. A duração do encontro seguiu no tempo esperado, em que os objetivos propostos para serem realizados foram alcançados.

\section{Alterações Realizadas a partir do Estudo Piloto 2}

No estudo piloto 2, foi testada a aplicabilidade do protocolo na versão grupal. Portanto, todas as sessões foram adaptadas para serem aplicadas em um grupo de adolescentes e em um grupo de pais, nas sessões separadas e em conjunto. As alterações no rapport, quando se referia aos adolescentes, foram passadas para o plural.

Observou-se que o grau de escolaridade em geral dos adolescentes que participaram do estudo piloto 2 era inferior ao do adolescente do estudo piloto 1 , assim como a capacidade de compreensão de alguns pais/responsáveis divergiu entre a etapa 1 e 2 do piloto, o que repercutiu na necessidade de que algumas intervenções propostas no formato individual fossem simplificadas no formato grupal; viu-se que o estímulo para a discussão da temática deveria ser realizado de forma mais oral do que escrita, lançando-se mão de recursos, como exemplos e explicações mais detalhadas sobre o que estava sendo proposto para ser discutido. A complementação com o material escrito auxiliou para que o que era verbalizado ficasse menos abstrato ao ser registrado no papel.

Contudo, alguns adolescentes demonstraram dificuldades no preenchimento de alguns recursos, como a planilha Prontidão para a Mudança, que aparece nas duas sessões com os adolescentes e serve para medir o quanto os adolescentes estão prontos para mudar seus comportamentos. Assim, foram melhoradas as instruções aos aplicadores para uma explicação mais esclarecedora do que estava sendo solicitado.

Nas sessões com os adolescentes, evidenciou-se a importância de explanar com o grupo alguns exemplos e a partir do que ia sendo trazido pelos adolescentes - eles foram preenchendo o material de estímulo. Istso porque, logo que eles receberam o material da sessão, demonstraram muita dificuldade em compreender o que estava sendo solicitado e se detiveram mais em cumprir com a tarefa do que em refletir sobre o exercício.

Para alguns adolescentes com baixa escolaridade, viu-se a importância de se desenvolver recursos adicionais mais ilustrativos e autoexplicativos. Como, por 
exemplo, incluir no exercício da Planilha das Vantagens e Desvantagens um material com duas colunas e com os desenhos de positivo e negativo indicando vantagens e desvantagens do uso, respectivamente.

No questionário Planilha de Gatilhos e Fissura, em que são listadas as razões para o uso de álcool e/ou outras drogas (tédio, fuga, relaxamento, socialização, melhoria da autoimagem, atração ou romance, "que se dane" e sem controle), observou-se a possibilidade de utilizar ilustrações, facilitando a identificação pelos adolescentes da(s) razão(ões) para o seu uso de drogas. No questionário Situações de Gatilhos ou Fissura, que traz algumas situações com potencial para virarem gatilhos para o uso, também foi vista a necessidade de ilustrar as situações referidas.

\section{Avaliação da Compreensão das Intervenções através do Pré-Teste e Pós-Teste}

Foi realizada a medida de pré e pós-teste para aferir se as intervenções propostas pelo protocolo estavam sendo compreendidas e, portanto, atingindo aquilo que se propunham. Para tanto, mediu-se a motivação para o tratamento por meio do grau de motivação e prontidão para mudança (Ladder), tanto no adolescente do estudo piloto 1 quanto no grupo de adolescentes do estudo piloto 2 .

Os adolescentes do estudo piloto 1 e estudo piloto 2 apresentaram uma tendência de aumento em sua motivação para mudar o comportamento de uso de drogas. $\mathrm{O}$ adolescente do primeiro estudo marcou no pré-teste $\mathrm{o}$ número quatro e no pós-teste o número cinco. Os adolescentes do estudo piloto 2 apresentaram no pré-teste uma média de 4,2 $(\mathrm{DP}=1,14)$ e, no pós-teste, a média foi 4,8 (DP=0,63). Essa diferença, não obstante, é apenas pontual, não sendo possível afirmar que as médias foram estatisticamente distintas.

\section{Discussão}

O objetivo deste estudo foi descrever o processo de tradução e adaptação para a realidade brasileira e adequação da aplicabilidade clínica e coletiva do Protocolo de Intervenção Breve proposto pelo Manual Brief Intervention for Adolescent Alcohol and Drug Use, que foi desenvolvido nos Estados Unidos. É importante ressaltar a relevância de se realizar esse processo em protocolos manualizados e testados, principalmente ao se tratar de um manual para tratamento do uso de drogas na adolescência com a abordagem familiar, algo relativamente novo dentro das modalidades de tratamento da área (Payá \& Figlie, 2010) que tem se mostrado um recurso terapêutico mais eficaz que o tratamento focado somente no adolescente (Robbins et al., 2011; Winters et al., 2012).

Conforme as etapas previstas na literatura (Hungerbühler \& Wang, 2016), o presente estudo não cumpriu com a fase de validação. Sendo assim, para verificar se a adaptação das intervenções estava de acordo com o instrumento original, deu-se ênfase às fases da revisão por especialistas do material traduzido e ao desenvolvimento de dois estudos-piloto que garantiram mais robustez à análise da adequabilidade, por serem utilizados com o público-alvo da intervenção (Barbosa, Miranda, \& Bueno, 2014; Beaton et al., 2000). Em se tratando dessas duas fases específicas, revisão por especialistas e os estudos-piloto, faz-se importante discutir alguns pontos.

A revisão por especialistas, neste estudo, também necessitou de alterações no processo de análise de conteúdo, em que se tem como item fundamental a medida de concordância de $80 \%$ entre os juízes (Pasquali, 2016). Tendo em vista que não se tratava de uma escala, o número de itens de todo o manual trouxe a impossibilidade de pontuar a concordância entre os especialistas. $\mathrm{O}$ estudo apenas seguiu as indicações da análise das equivalências: semântica (significado das palavras), idiomática (convenções sobre expressões locais), experimental (experiências cotidianas) e conceitual e de itens (significado cultural), (Hungerbühler \& Wang, 2016).

O número de participantes para o estudo piloto 2 foi estipulado conforme recomendado por Hungerbühler e Wang (2016), de 10 a 20 pessoas. Vale salientar que esse valor não foi critério no estudo piloto 1 , já que o cuidado pela seleção do participante foi mais rigoroso, ao contar com participantes (adolescente e pais) com boa capacidade de compreensão e bom nível de escolaridade. Além disso, a participação de ambos os pais também foi um diferencial exigido nessa escolha, pois se pode contemplar a visão da dupla. Todos esses critérios se aproximaram da realidade do estudo original (Winters et al., 2012), porém, são exceções quando se trata da realidade brasileira (Laranjeira, 2012).

O fato de todos os participantes serem do sexo masculino pode ser justificado pela frequência menor de adolescentes do sexo feminino estarem sendo atendidas no período da coleta no estudo piloto 1 e, no estudo piloto 2, por se tratar de um local de atendimento restrito a adolescentes do sexo masculino. Essa limitação no método pode ser justificada pelo percentual maior de adolescentes do sexo masculino envolvidos com drogas; embora, no que diz respeito ao uso de álcool, o percentual de meninas esteja equivalente ao de meninos (Laranjeira, 2012). Ainda assim, a realidade brasileira aponta para uma frequência maior de uso de drogas entre homens (Bastos \& Bertoni, 2014).

A apresentação dos resultados se deu em um formato qualitativo e descritivo, pois o objetivo foi apresentar as etapas de todo o processo, de modo que, quando feito qualitativamente, pode-se contemplar todas as modificações realizadas. A apresentação dos dados de forma qualitativa encontrou respaldo na literatura, pois, de acordo com Hungerbühler e Wang (2016), geralmente os resultados do estudo piloto são levantados por meio de uma avaliação qualitativa. 
A partir da realização do teste de aplicabilidade, por meio dos estudos-piloto, viu-se a necessidade de alterações importantes da versão original, que foi desenvolvida nos EUA e no contexto escolar (Winters et al., 2012). Neste estudo, o acesso à aplicação da intervenção breve em escolas foi mais restrito, vendo-se como possibilidades mais tangíveis ambulatórios e clínicas de internação especializadas. Isso porque a indicação pela escola de alunos que estavam fazendo uso de drogas e a realização da intervenção breve no mesmo espaço implicaria na exposição do adolescente.

Portanto, o nível de escolaridade dos participantes internados para tratamento do uso problemático de drogas delatou uma realidade e uma dificuldade não verificada no estudo original (Winters et al., 2012), embora muito encontrada no contexto brasileiro entre usuários problemáticos de drogas. No estudo de Galduróz et al. (2010), realizado nas capitais brasileiras, com 48.155 estudantes de ensino fundamental e médio de escolas públicas, verificou-se que a maioria $(36,3 \%)$ tinha entre 13 e 15 anos, porém $71,6 \%$ frequentavam ainda o ensino fundamental, com um percentual de $45,9 \%$ de reprovações.

O objetivo de desenvolver uma versão coletiva para o protocolo (originalmente elaborado para aplicação individual) se deu pelo contato com os locais de tratamento de adolescentes usuários de drogas, onde se observou a restrição de salas, de tempo e de profissionais para darem conta da demanda. Somado a isso, estão os altos índices de uso de drogas na adolescência (Laranjeira, 2012) e a classificação econômica brasileira que se encontra com um percentual maior entre as classes C-E (www.abep. org/criterio-brasil). A modalidade de tratamento grupal pode contribuir para essa realidade na medida em que traz a possibilidade de tratar um número maior de pessoas ao mesmo tempo e custando menos do que a modalidade individual (Sobell \& Sobell, 2013).

Quanto aos dados que apresentam, se a intervenção breve está atingindo aquilo que se propõe atingir, cabe salientar que este estudo não objetivou avaliar a eficácia do protocolo, e sim, utilizou uma medida de pré e pós-teste para demonstrar a compreensão das intervenções pelo adolescente do estudo piloto $1 \mathrm{e}$, posteriormente, a mesma medida foi empregada para verificar se a intervenção breve poderia ser aplicada coletivamente. A análise descritiva dos resultados, detendo-se no incremento no pós-teste, trouxe indícios de que o adolescente do estudo piloto 1 compreendeu a proposta do protocolo, e os adolescentes do estudo 2 se beneficiaram da intervenção, mesmo em sua versão grupal.

A partir do material apresentado e discutido, conclui-se que este estudo cumpriu com seu objetivo e apresentou um protocolo de intervenção adaptado para adolescentes brasileiros que estejam fazendo uso problemático de drogas. É importante salientar que foram seguidas as principais fases no processo de adaptação do manual, e que essa adaptação é uma condição importante para que a intervenção esteja próxima de uma prática do dia a dia (Winters, Tanner-Smith, Bresani, \& Meyers, 2014).

Porém, houve algumas limitações no processo, como não apresentar o índice de concordância dos juízes e a validação, pois, neste estudo, não foram encontradas formas de desenvolver esses procedimentos - ficando como sugestão para outros, que objetivem adaptar manuais, o uso desses recursos em algum formato a ser desenvolvido. No presente estudo, viu-se como possibilidade a substituição por outros procedimentos para que o processo não perdesse sua qualidade.

Uma sugestão é o desenvolvimento de um estudo para replicar o ensaio clínico randomizado realizado pelos autores do manual original, para verificar a eficácia desse protocolo. Neste estudo, não foi objetivado discutir sobre a eficácia da intervenção breve, mas entende-se que os resultados demonstram que a versão traduzida para o português se mostrou aplicável na realidade brasileira e em adolescentes do grupo clínico. Logo, é considerada uma versão final do manual que pode ser testada em outros estudos com a mesma amostra.

\section{Referências}

American Psychiatry Association. (2014). Manual Diagnóstico e Estatístico dos Transtornos Mentais: DSM5. (5 ed.) Porto Alegre: Artmed.

Amodei, N., Lamb, R. J. (2004). Convergent and concurrent validity of the Contemplation Ladder and URICA scales. Drug and Alcohol Dependence, 73, 301-306.

Arria, A. M., Mericle, A. A., Rallo, D., Moe, J., White, W. L., Winters, K. C., \& O’Connor, G. (2013). Integration of parenting skills education and interventions in addiction treatment. Journal of Addiction Medicine 7(1). doi: 10.1097/ADM.0b013e318270f7b0

Barbosa, D. L. F., Miranda, M. C., \& Bueno, O. F. A. (2014). Tradução e adaptação do PayAttention! - Um programa de treinamento dos processos da atenção para crianças. Psicologia Reflexão e Crítica, 27(4), 775-783. doi: 10.1590/1678-7153.201427418

Bastos, F. I., Bertoni, N. (2014). Pesquisa Nacional sobre o uso de crack: quem são os usuários de crack e/ou similares do Brasil? Quantos são nas capitais brasileiras? Rio de Janeiro, ICICT/FIOCRUZ.

Beaton, D. E., Bombardier, C., Guillemin, F., \& Ferraz, M. B. (2000). Guidelines for the process of cross-cultural adaptation of self-report measures. Spine, 25(24), 3186-91.

Dembo, R., Winters, K. C., Belenko, S., Karas, L. M., Gulledge, L., \& Wareham, J. (2014). Brief Intervention for Truant Youth Sexual Risk Behavior and Marijuana Use. Journal Child \& Adolescent Substance Abuse, 23(5), 318-333. doi: 10.1080/1067828X.2014.928116 
De Micheli, D., \& Formigoni, M. L. (2000). Screening of drug in a teenage Brazilian sample using the drug use screening inventory (DUSI). Addictive Behaviors, 25(5), 683-691.

De Micheli, D., \& Formigoni, M. L. (2002). Psychometric properties of the Brazilian version of the drug use screening inventory. Alcoholism: Clinical and Experimental Research, 26(10), 1523-1528.

Dimeff, L. A., Baer, J. S. Kivlahan, D. R., Marlatt, G. A. (2002). Alcoolismo entre estudantes universitários: Uma abordagem de redução de danos. Brief Alcohol Screening and Intervention for College Students (BASICS). São Paulo: Editora UNESP.

Faria, M. L. V.C. (2016). A avaliação da efetividade de um modelo de intervenção breve (método BASICS) para o uso de risco de álcool em estudantes do ensino médio (Tese de doutorado). Universidade Estadual Paulista "Júlio de Mesquita Filho", Faculdade de Medicina de Botucatu.

Figlie, N. B. (2004). Motivação para tratamento do alcoolismo (Tese de doutorado). Universidade Federal de São Paulo. São Paulo, S.P.

Figlie, N. B., Dunn, J., \& Laranjeira, R. (2004). Estrutura fatorial da StagesofChangeReadinessandTreatmentEagernessScale (SOCRATES) em dependentes de álcool tratados ambulatorialmente. Revista Brasileira de Psiquiatria, 26(2),91-99.

Galduróz, J. C. F., Sanchez, Z. V., Opaleye, E. S., Noto, A. R., Fonseca, A. M., Gomes, P. L. S., \& Carlini, E. A. (2010). Factors associated with heavy alcohol use among students in Brazilian capitals. Revista de Saúde Pública, 44(2).

Hungerbühler, I., \& Wang, Y. (2016). Aspectos transculturais na adaptação de instrumentos. Em C. Gorenstein, Y. Wang, \& I. Hungerbühler. Instrumentos de avaliação em saúde mental. Porto Alegre, RS: Artmed.

Kaynak, Ö., Winters, K. C., Cacciola, J., Kirby, K. C., \& Arria, A. M. (2014). Providing alcohol for underage youth: What messages should we be sending parents? Journal of Studies on Alcohol and Drugs, 75(4). doi: 10.15288/jsad.2014.75.590

Laranjeira, R. (Ed.). (2012). II Levantamento Nacional de Álcool e Drogas. Instituto Nacional de Políticas Públicas do Álcool e Outras Drogas, INPAD. Recuperado de http://www.inpad.org.br/index.php?option $=$ com_content\&view $=$ article\&id $=106$

Maisto, S. A, Chung, T. A., Cornelius, J. R., \& Martin, C. S. (2003). Factor Structure of the SOCRATES in a Clinical Sample of Adolescents. Psychology of Addictive Behaviors, 17(2), 98-107. doi: 10.1037/0893-164X.17.2.98

National Institute for Abuse. (2014). Principles of Adolescent Substance Use Disorder Treatment: A Research-Based Guide. EUA: National Institute on Drug Abuse - NIDA.

Payá, R., \& Figlie, N. B. (2010). Abordagem familiar em dependência química. Em N. F. Figlie, S. Bordin, \& R. Laranjeira. (Eds.). Aconselhamento em dependência química. São Paulo: Roca.

Pasquali, L. (2016). Princípios de elaboração de escalas. Em C. Gorenstein, Y. Wang, \& I. Hungerbühler. Instrumentos de avaliação em saúde mental. Porto Alegre, RS: Artmed.

Pupo, M. C., Ribeiro, M., \& Marques, A. C. P. R. (2012). Escalas de avaliação. Em M. Ribeiro \& R. Laranjeira. O tratamento do usuário de crack. Porto Alegre, RS: Artmed.

Robbins, M. S., Feaster, D. J., Horigian, V. E., Rohrbaugh, M., Shoham, V., ... \& Szapocznik, J. (2011). Brief Strategic Family Therapy versus Treatment as Usual: Results of a multisite randomized trial for susbtance using adolescents. Journal of consulting and clinical psychology, 79(6), 713-727. doi: 10.1037/a0025477

Silva, E. C., \& Tucci, A. M. (2015). Intervenção breve para redução do consumo de álcool e suas consequências em estudantes universitários brasileiros. Psicologia Reflexão e Crítica, 28(4), 728-736. doi: 10.1590/1678-7153.201528410

Sobell, L. C., \& Sobell, M. B. (2013). Terapia de grupo para transtorno por abuso de substâncias: abordagem cognitivo-comportamental motivacional. Porto Alegre, RS: Artmed.

Terra, M. B., Silveira, D. X., Moreira, T. C., Ferigolo, M., Mazoni, C., Arena, F. X., \& Barros, H. M. T. (2009). Convergent validation study of the Contemplation Ladder for application via telephone in tobacco users. Jornal Brasileiro de Psiquiatria, 58(3), 143-149. doi: 10.1590/ S0047-20852009000300001

Vanassche, S., Sodermans, A. K., Matthijs, K., \& Swicegood, G. (2014). The effects of family type, family relationships and parental role models on delinquency and alcohol use among Flemish adolescents. Journal of Child and Family Studies, 23(1), 128-143.

Wadolowski, M., Hutchinson, D., Bruno, R., Aiken, A., Najman, J. M., Kypri, K., Slade, T., McBride, N., \& Mattick, R. P. (2016). Parents who supply sips of alcohol in early adolescence: A prospective study of risk factors. American Academy of Pediatrics. doi: 10.1542/peds.2015-2611

Winters, K. C., Botzet, A., Fahnhorst, T., \& Leitten, W. (2006). Brief intervention for adolescent alcohol and drug use: Manual. Center for Adolescent Substance Abuse Research, University of Minnesota.

Winters, K. C., Fahnhorst, T., Botzet, A., Lee, S., \& Lalone, B. (2012). Brief intervention for drug abusing adolescents in a school setting: Outcomes and mediating factors. Journal of Substance Abuse Treatment, 42(3), 279-288. doi: 10.1016/j.jsat.2011.08.005

Winters, K. C., Lee, S., Botzet, A., Fahnhorst, T., \& Nicholson, A. (2014). One-year outcomes and mediators of a brief intervention for drug abusing adolescents. Psychology of Addictive Behaviors, 28(2), 464-474. doi: 10.1037/a0035041

Winters, K. C., \& Leitten, W. (2001). Brief intervention manual. Minneapolis: University of Minnesota.

Winters, K. C., \& Leitten, W. (2001). Brief intervention for drug-abusing adolescentes in a school setting. Psychology of Addictive Behaviors, 21(2), 249-254.

Winters, K. C., Leitten, W., Wagner, E., \& Tevyaw, T. O. (2007). Use of brief interventions for drug abusing teenagers within a middle and high school setting. Journal of School Health, 77(4), 196-206.

Winters, K. C., Tanner-Smith, E. E., Bresani, E., \& Meyers, K. (2014). Current advances in the treatment of adolescent drug use. Adolescent Health, Medicine and Therapeutics 5, 119-210. doi: 10.2147/AHMT.S48053

recebido em setembro de 2016 aprovado em agosto de 2017

\section{Sobre as autoras}

Fernanda Cerutti é Mestre e Doutora em Psicologia Clínica, PUCRS, Docente do Curso de Graduação em Psicologia da IMED.

Bruna Fernandes da Rocha é Mestranda Programa de Pós-Graduação em Gerontologia Biomédica da PUCRS.

Luisa Raquel Bridi Dacroce é Graduanda de Psicologia, Bolsista de Iniciação Científica, PUCRS.

Irani Iracema de Lima Argimon é Docente Titular do PPG em Psicologia, PUCRS, Bolsista Produtividade CNPq - 1D. 